\title{
«À ciel ouvert » de Nelly Arcan : une réécriture du mythe de Pygmalion et Galatée
}

\author{
Ghada Saber Mohamed \\ Maître de conférences \\ Département de français, faculté de Pédagogie, université d'Ain Chams
}

\begin{abstract}
«À Ciel ouvert» de Nelly Arcan : une réécriture du mythe de Pygmalion et Galatée
\end{abstract}

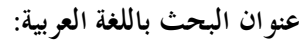

$$
\begin{aligned}
& \text { إعادة كتابة أسطورة بيجماليون وجالاتيا في رواية "في الهواء الطلق" للكاتبة نيللي أركان } \\
& \text { عنوان البحث باللغة الانجليزية: }
\end{aligned}
$$

The Rewriting of Pygmalion and Galatea's Myth in Nelly Arcan's " A Ciel ouvert"

\begin{abstract}
Par le biais de l'approche mythocritique et selon les trois critères d'« émergence », de «flexibilité » et d'《irradiation» établis par Brunel, nous essayons, dans un premier temps, de repérer les éléments ou occurrences mythiques qui se trouvent dans l'hypertexte arcanien et qui aident à l'émergence du mythe de Pygmalion et Galatée dans notre corpus. Dans un deuxième temps, l'accent est mis sur la flexibilité de la matière mythique, à savoir la manière dont les figures mythiques de Pygmalion et de Galatée se greffent sur les personnages du roman ainsi que les différentes formes de modulations auxquelles elles se prêtent et leur signification. Dans un troisième et dernier temps, nous essayons de montrer le pouvoir de rayonnement et d'irradiation du mythe dans l'œuvre de Nelly Arcan.

L'étude des modalités de la réécriture du mythe de Pygmalion et Galatée ainsi que son réinvestissement significatif dans le roman contemporain $\grave{A}$ ciel ouvert nous a révélé comment Nelly Arcan a réussi à re-créer de nouvelles représentations des rôles traditionnels du masculin et du féminin en faveur d'un rééquilibrage des rapports de pouvoir entre les sexes.
\end{abstract}

\section{Abstract}

By means of the mythological critical approach and according to the three criteria of "emergence", "flexibility" and "radiance" established by Pierre Brunel, we try, at first, to show mythical elements or occurrences which are in Nelly Arcan's hypertext and which help in the emergence of the myth of Pygmalion and Galatea in A ciel ouvert. Second, we study the flexibility of the mythical matter, namely the way the mythical figures of Pygmalion and Galatea are incarnated the characters of the novel as well as the various forms of their changes and their meaning. Third and finally, we try to show the myth power of radiance in Nelly Arcan's novels.

The study of the rewriting of Pygmalion and Galatea's myth and its significant reinvestment in $\dot{A}$ ciel ouvert reveals to us how Arcan manages to 
recreate new representations of man/woman traditional roles in order to rebalance the power relations between them.

$$
\begin{aligned}
& \text { هتم هذه الدراسة بتحليل مظاهر وأساليب إعادة كتابة أسطورة بيجماليون القديمة في رواية "في الهواء الطلق" للكاتبة }
\end{aligned}
$$

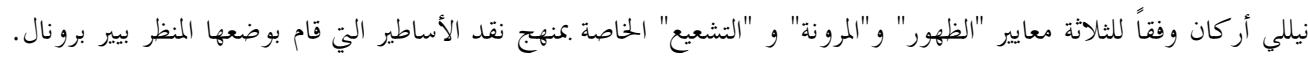

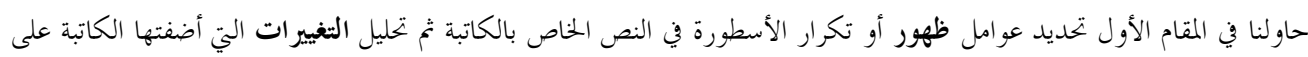

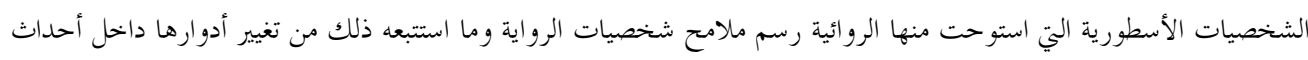

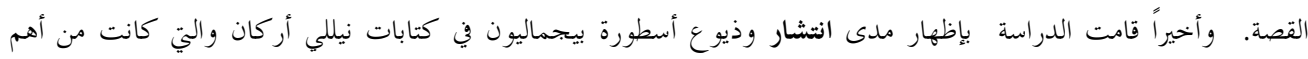

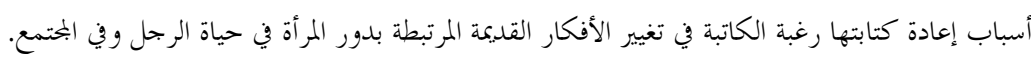

Dans l'introduction de son ouvrage intitulé Mythocritique : théorie et parcours, Pierre Brunel déclare qu'un regard différent peut être porté sur les textes littéraires si on considère avec une attention plus soutenue les éléments mythiques qu'ils contiennent ${ }^{1}$. De cette conviction est née la présente étude.

En effet, le XXe siècle a vu naître une constellation d'écrivains qui se sont inspirés des grands mythes anciens. Réécrire des mythes qui tiennent un discours sur les identités féminine et masculine reste encore la voie royale empruntée par de nombreuses romancières contemporaines, telles que Sylvie Germain, Amélie Nothomb, Assia Djebar et Marie-Sissi Labrèche, entre autres, qui nous invitent à relire autrement les grands textes fondateurs de la culture occidentale.

À l'instar de ces écrivaines, la romancière québécoise Nelly Arcan adopte dans ses œuvres des stratégies de réécriture des mythes antiques célèbres et qui lui sont propres. À titre d'exemple, son troisième roman $\dot{A}$ ciel ouvert (2007) propose une reprise de grandes figures mythiques grecques, celles de Pygmalion et de Galatée, celle de Méduse et celle des Amazones, non liées les unes aux autres dans la mythologie grecque. Arcan réécrit leurs mythes pour leur donner une nouvelle dimension, en lien avec notre époque, où le rapport homme/femme sera repensé.

C'est du concept de l'hypertextualité de Gérard Genette que l'élaboration du roman s'inspire, l'hypertextualité étant " toute relation unissant un texte $B$ [1'hypertexte] à un texte antérieur $A$ [1'hypotexte] sur lequel il se greffe d'une manière qui n'est pas celle du commentaire» ${ }^{2}$.

Ainsi le mythe lui-même sera considéré selon la terminologie de Genette comme un "hypotexte» alors que le récit de $\dot{A}$ ciel ouvert, (désormais $A C O$ ), comme un « hypertexte » par rapport au schéma mythique.

Dans le but de restreindre l'objet de notre travail, nous nous attarderons uniquement au mythe de Pygmalion et Galatée qui constituera l'essentiel de nos analyses, étant donné son importance et son ascendance au texte fictionnel arcanien.

Notons de prime abord que la légende de Pygmalion et Galatée a incessamment inspiré les artistes et les œuvres d'art de tout temps, de tout pays, dans tous les domaines : en littérature ${ }^{3}$, en peinture, en sculpture, en musique, au théâtre et au cinéma.

Notre problématique sera, par conséquent, la suivante :

Comment Nelly Arcan, dans sa réécriture du mythe de Pygmalion et Galatée et à travers la greffe de ces figures mythiques sur ses personnages appartenant à l'époque moderne, a-t-elle réussi à re-créer de nouvelles représentations des rôles traditionnels du masculin et du féminin? 
Dans cet article, il s'agira donc, par le biais de l'approche mythocritique ${ }^{4}$, d'étudier notamment les modalités de réécriture du mythe de Pygmalion et Galatée ainsi que son réinvestissement significatif dans le roman $A C O$, selon les trois critères $d$ ' « émergence », de «flexibilité » et d'《irradiation» établis par Pierre Brunel $^{5}$.

C'est pourquoi notre plan se présentera comme suit :

Dans un premier temps, nous essayerons de repérer les éléments ou occurrences mythiques qui se trouvent dans l'hypertexte arcanien. Il s'agit ainsi de l'émergence du mythe de Pygmalion et Galatée.

Dans un deuxième temps, l'accent sera mis sur la flexibilité de la matière mythique, à savoir la manière dont les figures mythiques de Pygmalion et de Galatée se greffent sur les personnages du roman ainsi que les différentes formes de modulations auxquelles elles se prêtent et leur signification.

Dans un troisième et dernier temps, nous essayerons de montrer le pouvoir de rayonnement et d'irradiation du mythe dans l'œuvre $A C O$ de Nelly Arcan comme nous chercherons à voir si cette irradiation s'est étendue à d'autres écrits de l'écrivaine.

A ciel ouvert relate l'histoire de Rose Dubois, une jeune femme d'une trentaine d'années habitant le Plateau Mont-Royal, un quartier de Montréal, avec son fiancé Charles Nadeau. Alors qu'ils vivaient en parfaite harmonie, la rencontre avec Julie O'brien, leur nouvelle voisine, va changer cette situation. En effet, Charles se trouve tellement épris des charmes de Julie qu'une liaison amoureuse les attache l'un à l'autre.

Devenues ennemies redoutables, les deux femmes rivalisent pour s'emparer de l'homme, chacune à sa manière. Or, Charles choisit enfin de partir avec Julie laissant Rose à son désespoir. Vaincue, celle-ci trouve refuge auprès d'un chirurgien esthétique, Dr Gagnon, qui l'aidera à mettre à exécution un stratagème, dans l'espoir de regagner le cœur de son ancien amant. Rose décide de subir une vaginoplastie $^{6}$ pour offrir à Charles le corps juvénile dont il rêvait.

Avant de commencer la première partie de notre article, il convient peut-être de présenter un rappel du mythe originel de Pygmalion et Galatée repris dans $\grave{A}$ ciel ouvert.

\section{Mythe de Pygmalion et Galatée:}

Dans la mythologie grecque, Pygmalion était un sculpteur très renommé dont les belles œuvres émerveillaient les femmes. Celles-ci rêvaient toutes d'être un jour la femme du roi.

Quant à Pygmalion, et d'après Ovide dans ses Métamorphoses ${ }^{7}$, il déteste et fuit le sexe féminin enclin par sa nature portée au vice. Refusant toujours d'avoir une compagne, il se voue alors au célibat et à la sculpture. C'est ainsi que l'artiste passe son temps, seul, enfermé dans son atelier, afin d'achever ses œuvres.

Un jour l'envie le prend de sculpter, dans l'ivoire, une statue représentant son idéal de femme parfaite qui porte tous ses vœux. Une fois achevée, la statue paraît bien réussie, douce, rayonnante, d'une beauté incomparable. De jour en jour le sculpteur s'applique à la peaufiner jusqu'à sa perfection.

La contemplant jour et nuit, Pygmalion finit par tomber amoureux de son œuvre, l'imaginant être une belle vierge en chair et en os. Ainsi, il lui parle, la 
couvre de baisers pleins d'amour, lui prodigue de tendres caresses. Il se plaît également à la parer de riches habits et bijoux et la couche même à ses côtés.

Le jour de la fête d'Aphrodite, Pygmalion implore la déesse de l'amour de lui donner une épouse aussi belle et aussi parfaite que la vierge d'ivoire. En réponse à ses prières, Aphrodite insuffle la vie à sa statue. Le sculpteur qui voit son œuvre devenir une femme vivante finit par l'épouser.

Dans cette première partie de notre étude, comme noté plus haut, nous chercherons à étudier les manifestations de l'émergence du mythe de Pygmalion et Galatée dans le texte $\dot{A}$ ciel ouvert ainsi que les mythèmes ${ }^{8}$ concernant l'essentiel du mythe et réutilisés par Arcan dans son hypertexte.

I) L'ÉMERGENCE DU MYTHE:

Rappelons que l'émergence, selon les lois de la mythocritique, est tout ce qui aide à reconnaître la présence du mythe au sein du récit. Celle-ci peut prendre la forme d'un nom propre ou d'un objet, d'un lieu, d'un événement, d'un acte fondamental ou même d'une caractéristique en rapport avec le mythe.

À la première lecture du roman, les signes visibles du mythe de Pygmalion et Galatée paraissent rares. Autrement dit, la présence du mythe demeure latente, car jamais explicitement mentionné. Toutefois, une lecture approfondie du roman montre que les traces de la réécriture du mythe se révèlent dans certaines caractéristiques et certains rôles communs aux personnages du récit et aux figures mythiques.

En effet, dans $\grave{A}$ ciel ouvert, deux personnages masculins réincarnent Pygmalion le sculpteur: Charles Nadeau et le docteur Marc Gagnon. En outre, le mythe de Pygmalion qui se forme sous la plume arcanienne traverse également le texte grâce aux personnages féminins de Rose et de Julie qui semblent être les reflets d'une seule et même figure mythique, celle de Galatée.

Il s'agira ici de mettre en perspective les représentations de ce mythe qui se trouvent dans l'hypertexte arcanien avec ses éléments fonctionnels minimaux, à savoir ses mythèmes fondamentaux. Deux de ces mythèmes sont réutilisés par Nelly Arcan dans son récit fictionnel : le mythème de l'homme créateur/dominant et celui de la femme objet/statue parfaite.

\section{1- Mythème de l'homme créateur/dominant}

Le mythe de Pygmalion suscite généralement le thème de l'homme créateur qui non seulement crée de sa main la femme, mais lui octroie également sa féminité en lui attribuant la vie. Force est de constater également que l'une des prémisses du mythe est la quête de la beauté idéale.

Ainsi, dans $A C O$, Charles, photographe de mode, crée des «Galatée virtuelles » par la technique de Photoshop, «l'outil principal du sculpteur moderne $q u$ 'il incarne $»^{9}$. Par ce biais, il élimine tous les défauts physiques et les impuretés physionomiques des corps photographiés puis rectifiés et retouchés sur un écran d'ordinateur avant d'être exposés sur les affiches publicitaires ou les pages des revues de mode.

Enfin, à l'image du sculpteur perfectionnant sa statue d'ivoire, Charles, par le truchement de l'appareil photographique, "crée le canon, la norme à respecter en matière de beauté féminine $\gg{ }^{10}$. 
Le deuxième type de Pygmalion dans le texte d'Arcan, le docteur Gagnon, est un chirurgien plastique ${ }^{11}$ dont le métier consiste à sculpter des Galatée réelles. Ce Pygmalion s'applique à façonner, « corriger » ou remodeler l'apparence ou les corps des femmes, conformément aux canons esthétiques instaurés par l'industrie de la mode.

D'ailleurs, le corps féminin reconstruit par ce chirurgien devient une œuvre d'art à laquelle il appose sa signature " pour en réclamer le droit d'auteur $\rangle^{12} \mathrm{ou}$ pour confirmer que le «travail vient d'une même main », $(\mathrm{p} .120)$.

Pour la circonstance, la profession du personnage arcanien confirme ellemême l'hiérarchie dans les rôles masculins/féminins qui se trouvent dans le mythe, puisque le destinataire de chirurgie esthétique typique, l'objet à opérer, est le plus généralement une femme alors que le chirurgien, le destinateur, est presque toujours un homme.

Photographe ou chirurgien, tout comme Pygmalion, chacun gardera toute sa puissance et sa dominance sur la femme. Au demeurant, s'ils sont amenés à porter sur elle un regard morcelé c'est qu'ils l'appréhendent toujours en tant que parties d'un corps, jamais comme sujet unifié.

\section{2- Mythème de la femme objet / statue parfaite:}

Notons ici que le mythe n'accorde aucune importance à la subjectivité féminine. En d'autres termes, quand Aphrodite, la déesse de l'amour, a exaucé le vœu de Pygmalion et qu'elle a transformé sa statue en femme en chair et en os, Galatée demeure traitée comme un objet d'art. Ses sentiments et ses émotions ne sont jamais rapportés ni décrits par le mythe.

Par ailleurs, la passion de Pygmalion pour son œuvre repose essentiellement et uniquement sur ses traits physiques. Statue inanimée, Galatée, création de Pygmalion, n'est qu'un objet réifié, objet passif qui subit les actes de son créateur. En somme, cette figure mythique est reconnue comme entièrement possédée par l'homme qui l'a taillée et non comme sujet pensant ou agissant ${ }^{13}$.

Dans $\dot{A}$ ciel ouvert, cette hiérarchie dans les rôles, où le masculin est lié à la créativité et à la dominance tandis que le féminin se trouve du côté de la passivité et de l'esclavage, reconduit la thématique du sujet/objet maintenue par Arcan dans sa réécriture du mythe de Pygmalion et Galatée.

Ainsi, les deux jeunes protagonistes féminines occupent en l'occurrence le rôle de la femme-statue qui demeure sans le moindre pouvoir face à son amour. D'un côté, Rose est présentée, dès le début du récit, comme un être inférieur, docile, toujours victime de la tyrannie de son amant pour lequel elle est prête même au sacrifice de soi.

C'est pourquoi lors de sa première rencontre avec Charles au «shooting» d'une publicité, bien qu'elle ait deviné les goûts sexuels pervers de l'homme, Rose choisit de s'engager dans une liaison avec lui.

Durant les cinq années de leur engagement, la préoccupation à l'égard du désir de Rose et son assouvissement n'habite Charles que pendant la première année uniquement. Au fil du temps, le plaisir sexuel de celui-ci ne s'épanouit qu'à travers des actes malsains qui annihilent dans un premier temps le corps de Rose puis dans un deuxième temps ses besoins en tant que femme. 
À titre d'exemple, sadique, Charles ne ressent du plaisir qu'à la vue du corps féminin réduit à son état de réification ou de victimisation, soit un corps blessé, ecchymosé ou cicatrisé, opéré, mutilé, abîmé ou même meurtri. Ce sont en outre les séquences de vidéo « de tous ces morceaux qui le faisaient haleter », (p.112).

Manipulée par le personnage masculin qui n'a de considération que pour son autosatisfaction, Rose se consent en silence à tous les délires pornographiques de son homme et finit par se placer d'elle-même «en état de pur fétiche fait pour le sexe sans sexe $»,(\mathrm{p} .107)$.

Ainsi, à un moment donné, la passivité de Rose prend la forme d'une abnégation de soi qui la mène à transgresser certaines limites morales, psychologiques et physiologiques pour plaire à Charles.

De son côté, Julie, sa rivale, accepte elle aussi les scénarios érotiques de Charles où elle joue le rôle de la femme/statue. Le plaisir charnel sera presqu'absent de sa relation intime avec Charles où elle adopte une «immobilité réglementaire, les yeux fermés, passive, [comme] un cadavre», (p.194). Elle va quelquefois jusqu'à l'automutilation par coups de rasoir pour combler le désir de son partenaire.

De ce fait, dans leurs rapports avec Charles, Rose et Julie sont réduites à des corps/objets au service du sujet mâle, à des êtres qui s'abîment, physiquement et psychologiquement, pour assouvir les besoins ou arracher la jouissance sexuelle de leur amant.

Le texte arcanien semble dénoncer cette conception de la féminité selon laquelle la femme/statue ne gagne sa légitime existence qu'aux côtés de l'homme. Ne lui suffisait-il pas de lui satisfaire ses désirs d'ordre sexuel aux dépens des siens?

C'est pourquoi Rose et Julie sont toutes les deux obnubilées par l'image de la femme physiquement parfaite et qu'elles chercheront à se construire. Recourant à la chirurgie plastique, aux sévices gymnastiques et diététiques, aux productions cosmétiques, elles choisissent sciemment diverses procédures pour «statufier» (p.62) leurs corps et leurs traits, conformément aux normes esthétiques modernes.

$\mathrm{Ne}$ serait-ce pas un nouvel aspect d'asservissement infligé à ces femmes/Galatée devenues victimes de leur « acharnement esthétique » (p.89) qui les claustre dans l'idée d'objectivation ou plus précisément d'uniformisation corporelle?

[Rose] était vraiment belle mais d'une façon commerciale, industrielle », l'avait noté Julie, "sans la juger puisqu'elle en faisait elle-même partie de cette famille de femmes dédoublées des affiches, (p.15).

Dans cette dynamique d'esclavage, c'est le brouillage des limites entre le noble et le trivial, le naturel et l'artificiel, la réalisation et la réduction à néant du moi qui va placer les protagonistes féminines dans une position de précarité, voire de danger qui va changer l'image de la figure mythique de Galatée et changer par la suite les rôles des deux héroïnes.

C'est ce que nous allons étudier dans cette deuxième partie de notre travail en nous focalisant sur la flexibilité de la matière mythique. 


\section{2- LA FLEXIBILITÉ DE LA MATIÈRE MYTHIQUE:}

Comme l'indique son nom, la flexibilité désigne "la souplesse» avec laquelle Arcan adapte les éléments mythiques à son texte fictionnel et «les modulations surtout dont ce texte lui-même est fait» ${ }^{14}$.

Il s'agira pour nous d'analyser les différentes variations et transformations apportées aux figures mythiques au sein du récit arcanien, permettant ainsi à l'auteure d'établir une certaine distance entre le texte mythique et son propre texte.

En effet, dans $A C O$, Nelly Arcan subvertit certains éléments du mythe de Pygmalion par la mise en œuvre des deux stratégies d'écriture ou de réécriture que sont le dédoublement des figures mythiques représentées dans son récit et le renversement des mythèmes ou des rôles inhérents à ces figures dont l'auteure s'est inspirée.

\section{a) Dédoublement :}

Cette première stratégie se révèle dans les modulations que Nelly Arcan apporte à la structure du mythe par la création d'une image double aux figures mythiques.

Nous savons déjà qu'elle confère à deux personnages masculins (Charles et Dr Gagnon) le rôle de Pygmalion et aux deux héroïnes (Rose et Julie) le rôle de Galatée, tout en ancrant les deux figures mythiques dans le contexte contemporain de sa fiction.

Ainsi, dans l'ère contemporaine, Pygmalion a pour métier la photographie ou la chirurgie esthétique. Quant à Galatée, elle est styliste ou réalisatrice de documentaires. C'est surtout une femme dont le corps est sculpté réellement à coups de scalpel ou virtuellement par des retouches numériques.

Cette stratégie provoque, à notre sens, la diffraction de la figure mythique qui contribue à ajouter de nouvelles strates de signification au sens premier du mythe envahissant l'hypertexte arcanien.

D'une part, le dédoublement de la figure mythique de Pygmalion en Charles et Dr Gagnon s'attache à faire de ces personnages masculins une représentation de tous les hommes qui, derrière leur vision machiste et leur désir de fragmentation de la figure féminine cherchent à cacher une peur ou un refus de reconnaître la femme en tant que sujet autonome ou indépendant.

Par contre, la figure de Galatée se trouve d'autre part enrichie du traitement qu'en fait Nelly Arcan. C'est la mise en scène des personnages féminins de Rose et de Julie donnant deux visions concurrentes de Galatée qui constitue, à notre avis, un des aspects les plus importants de la flexibilité de la matière mythique réutilisée dans le roman.

En effet, si Arcan choisit ces deux femmes pour incarner, toutes les deux, la figure de Galatée, c'est parce que

[p]hysiquement, elles se ressemblaient, c'est vrai, mais cette ressemblance en indiquait une autre, cachée derrière, celle de leur mode de vie consacré à se donner ce que la nature leur avait refusé; [...] abandons d'ellesmêmes mises en pièces par la technique médicale, par son talent de refonte. Elles étaient belles de cette volonté féroce de l'être, (pp.15-16). 
Nonobstant cette ressemblance, les personnages féminins d'À ciel ouvert restent des rivales, de caractère et de vision différents. C'est pourquoi, à un certain moment du récit, chacune suivra un parcours différent, marquant par là l'évolution de la figure de Galatée.

C'est ce qui représente, en fait, une première rupture dans la réécriture du mythe marquée par le renversement de ses mythèmes et par conséquent le détournement des rôles des protagonistes.

\section{b) Renversement :}

Il s'agit ici de discerner les différentes transformations que subit le mythe de Pygmalion et Galatée qui, par sa flexibilité, permet au texte du roman de sortir du cadre fixe de l'hypotexte ancien ou du récit habituel qui avait marqué depuis toujours l'imaginaire occidental.

Dans notre corpus, et contrairement au récit mythique, la primordialité des rôles des Pygmalion en tant que sujet dominant va décliner alors que celui de Rose et de Julie - Galatée - devient lui-même le moteur essentiel de l'action. C'est cette figure féminine, dès lors centrale, qu'Arcan nous invite à suivre dans le processus de son évolution.

Tout compte fait, la façon dont les personnages se développent par rapport aux événements du roman participe à la modification des mythèmes propres au mythe. À titre d'exemple, un mythème comme celui de l'homme créateur/dominant va disparaître. Par conséquent, Pygmalion ne reste plus à même de conserver constamment Galatée telle qu'il la désire dans le texte contemporain d'Arcan.

Quant à Galatée, elle sera dotée d'une certaine autonomie acquise, d'une tendance à refuser le rôle d'objet passif qui lui était traditionnellement attribué dans le mythe ancien. C'est ainsi que paraît l'un des points forts de la réécriture du mythe chez Arcan.

En fait, c'est à la faveur de l'infléchissement de l'hypotexte mythique, c'està -dire le renversement des rôles des protagonistes, qu'on assistera à la transformation totale des rapports de force entre la femme objet/statue et l'homme créateur/dominant conférant à la figure féminine un nouveau rôle actif.

Quel est donc ce nouveau rôle concernant Rose et Julie?

Pour sa part, Julie, consciente d'elle-même et de ses situations, elle va "penser sa propre aliénation »", cesser de jouer le rôle de femme statue/objet tel qu'elle l'avait pratiqué au début du roman. Refusant de basculer de plus en plus dans l'abjection érotique, elle choisit d'abandonner définitivement Charles. Elle quitte ainsi son rôle de Galatée en détournant l'image de la femme faite pour le désir.

Quant au second renversement, il concerne le rôle que joue Rose dans le texte. L'autodénigrement continu de son corps et la perte de son pouvoir de séduction pour Charles l'avaient poussée hors des frontières du soi, hors des limites et des interdits.

Animée par un profond désir de vengeance après que son ex-amant l'a trahie, elle décide de subir une vaginoplastie qui constitue, selon elle, une forme de prise de pouvoir. Ce changement important de sa personnalité annonce déjà l'éclatement du canon de la figure mythique de Galatée, figure passive et effacée au profit de celle de Pygmalion. 
Plus le récit avance plus Rose acquiert une force d'action dont elle a été longtemps privée. À un certain moment du récit, la Galatée/objet qu'elle fut au début de l'histoire renverse le pouvoir des Pygmalion (Charles et Dr Gagnon), défiant ainsi l'hégémonie des créateurs de sa « statue ».

Pour Dr Gagnon devenu fou amoureux de Rose, elle réussit à le manipuler, lui dictant la façon dont elle veut se remodeler :

Elle savait que Marc l'opérerait en temps voulu, qu'il le

ferait parce qu'il l'aimait, elle savait que, pour elle, il irait

contre sa propre volonté, au bout d'une folie qui n'était

pas encore la sienne, mais qui le deviendrait, (p.174).

C'est juste après l'opération que le chirurgien/Pygmalion commence à perdre tout son pouvoir sur l' « œuvre » qu'il a sculptée. Rejeté par Rose, le docteur Gagnon s'apparentera aux Pygmalion dépassés puis abandonnés par l'ouvrage de leurs propres mains. À partir de là, dans le processus de la réécriture du mythe, son rôle touche à sa fin.

Quant à Charles/Pygmalion, le second personnage masculin, Rose renverse son pouvoir en s'arrogeant elle-même la suprématie des rôles à jouer. Un ultime renversement du mythe de Pygmalion dans l'hypertexte arcanien figurera en fait dans le dernier chapitre du roman constituant la clôture du mythe réécrit par l'auteure.

Contrairement à Pygmalion mythique, Charles Nadeau, le photographe, ne connaîtra pas une fin heureuse ${ }^{16}$. Dans ce dernier épisode, il perd toute sa puissance et paie le prix de son omnipotence et sa perversité qui ont contribué à transformer Rose en une nouvelle Galatée.

Ainsi, à la rencontre de Rose, Charles va prendre conscience de la monstruosité de ses goûts anormaux et de ses actes déviants qui, étant « le contraire de [...] la nature », "l'horrifiaient lui-même », (p. 106). Devenant lucide, il ne tardera pas par ailleurs à entrer dans un délire avant de se jeter du toit de l'immeuble où il habite.

Ayant traité la question de l'émergence et de la flexibilité, reste à montrer le pouvoir du rayonnement du mythe de Pygmalion et Galatée dans le roman de Nelly Arcan ainsi que l'irradiation de la matière mythique à travers son écriture.

\section{3) L'IRRADIATION DU MYTHE}

Selon la loi mythocritique, nous tenterons ici de saisir l'intensité du rayonnement du mythe de Pygmalion et Galatée et de ses mythèmes dans l'écriture de Nelly Arcan.

Rappelons que, selon Brunel, «l'élément mythique, même s'il est ténu, même s'il est latent, doit avoir un pouvoir d'irradiation. Et s'il peut se produire une destruction, elle ne sera que le résultat de cette irradiation ${ }^{17}$. De même, il cite deux sources d'irradiation sous-textuelle. La première est l'ensemble de l'œuvre de l'écrivain. Une figure mythique, présente dans un texte de cet écrivain, peut donc rayonner dans un autre texte où elle n'est pas explicite.

La deuxième source d'irradiation est le mythe lui-même et son « inévitable rayonnement » dans la mémoire et dans l'imaginaire de l'écrivain qui n'a même pas besoin le plus souvent de le rendre explicite. 
À notre sens, l'irradiation du mythe est généralement difficile à nier quand ce dernier est mis en valeur par l'auteur lui-même, comme c'est déjà étudié dans notre corpus, et plus généralement lorsqu'il ne cesse de se manifester de façon plus ou moins évidente dans ses textes.

Dans les œuvres d'Arcan, le mythe de Pygmalion et Galatée s'irradie ainsi par les différentes reprises qu'en fait l'auteure ${ }^{18}$. C'est ce qui nous permet en fait de reconnaitre l'importance majeure de cette légende au centre de la démarche créatrice de l'écrivaine.

En guise de conclusion, nous pouvons dire que le roman $A C O$ garde son appartenance au mythe de Pygmalion pour tout lecteur attentif au texte.

Pourtant, chez Arcan, l'originalité de la réécriture du mythe réside non seulement dans sa présence latente qui sous-tend le roman mais surtout dans la manière dont Nelly Arcan modifie la matière mythique selon sa propre vision.

En effet, les stratégies de réécriture propres à Arcan que sont le dédoublement et le renversement ont contribué à réactualiser le mythe originel dans le contexte contemporain de son roman, tout en y ajoutant de nouvelles significations en lien avec notre époque.

Enfin, grâce a la subversion des rôles des figures mythiques qui se greffent sur ses personnages, l'auteure a pu redéfinir les rapports de force homme/femme dans un souci de rééquilibrage des positions de pouvoir des sexes et plus précisément en faveur d'une réhabilitation du personnage féminin. 


\section{Bibliographie:}

\section{Corpus}

- ARCAN Nelly, À ciel ouvert, Paris, Éditions du Seuil, 2007.

Ouvrages de critique et théories

- BRUNEL Pierre, Mythocritique. Théorie et parcours, Paris, Presses universitaires de France, 1992.

- DUGAS Marie-Claude, (en ligne), Corps, identité et féminité chez Nelly Arcan et Marie-Sissi Labrèche, mémoire de maîtrise, Montréal, Université de Montréal, 2010.

- GENETTE Gérard, Palimpsestes. La Littérature au second degré, Paris, Éditions du Seuil, Points essais, 1992.

- JOUBI Pascale, (en ligne), Mythes et monstres dans Folle et À ciel ouvert de Nelly Arcan, mémoire de maîtrise, Montréal, Université de Montréal, 2014.

- MONNEYRON Frédéric, THOMAS Joël, Mythes et littérature, Paris, Presses Universitaires de France, 2002.

Articles de périodiques en ligne

- ABDELMOUMEN Mélikah, Liberté, féminité, fatalité, cyberentretien avec Nelly Arcan (en ligne), Spirale, no.215, 2007, pp. 34-37.

- JOUBI Pascale, «Pygmalions contemporains ou la nouvelle Galatée dans $\grave{A}$ ciel ouvert de Nelly Arcan » in Analyses, vol.11, n², printemps-été 2016, pp. 140-154.

Sites d'Internet

- https://agoraclass.fltr.ucl.ac.be/concordances/ovideX/lecture/7.htm

- https://papyrus.bib.umontreal.ca/xmlui/handle/1866/4753

- https://papyrus.bib.umontreal.ca/xmlui/handle/1866/11956 


\section{Les références:}

1 Pierre BRUNEL, Mythocritique. Théorie et parcours, Paris, Presses universitaires de France, 1992, p. 11.

2 Gérard Genette, Palimpsestes. La Littérature au second degré, Paris, Éditions du Seuil, Points essais, 1992, p.13.

3 Nous citons à titre d'exemple en littérature: Pygmalion (1770), scène de Jean-Jacques Rousseau, "Pygmalion et Galatée », poème de Charles Cros paru dans son recueil posthume Le collier de griffes (1908), Pygmalion (1914), pièce de l'irlandais Georges Bernard Show, Le deuxième Sexe, roman de Simone de Beauvoir (1949).

4 Pierre Brunel conçoit la mythocritique comme un mode d'analyse littéraire qui «s'intéressera surtout à l'analogie qui peut exister entre la structure du mythe et la structure du texte», op.cit., p.67.

5 Ibid., pp.72-86.

6 La vaginoplastie est une opération de chirurgie plastique reconstructive, qui consiste à rétrécir l'orifice vaginal.

7 Il s'agit d'un long poème épique écrit par le poète latin Ovide vers l'an I ou II. Cette œuvre majeure composée de 15 livres raconte l'histoire d'environ 250 mythes du monde, dont celui de Pygmalion (livre X), en 12000 vers approximativement.

8 Par la reprise du concept de mythème forgé par Lévi-Strauss, Frédéric Monneyron et Joël Thomas définissent le mythème comme " une courte séquence fonctionnant en tant qu'une unité autonome, et en même temps reliée à un système mythique plus vaste». C.f. Mythes et littérature, Paris, Presses Universitaires de France, 2002, p. 71.

9 Pascale JOUBI, «Pygmalions contemporains ou la nouvelle Galatée dans À ciel ouvert de Nelly Arcan » in Analyses, vol.11, n², printemps-été 2016, p.143.

10 Marie-Claude DUGAS, Corps, identité et féminité chez Nelly Arcan et Marie-Sissi Labrèche, mémoire de maîtrise, Montréal, Université de Montréal, 2010, p.48.

11 Notons qu'étymologiquement du grec le mot «plastique» signifie ce qui est relatif au « modelage », tout ce qui peut être « façonné ».

12 Pascale JOUBI, Mythes et monstres dans Folle et À ciel ouvert de Nelly Arcan, mémoire de maîtrise, Montréal, Université de Montréal, 2014, p.83.

13 D'après Ovide, la seule "réaction » attribuée à Galatée lors de sa recréation, c'est de " rougir » avant de «lev[er] timidement son regard» pour voir « en même temps que le ciel [...] celui qui l'aimait».

14 Pierre BRUNEL, op.cit., p.77.

15 C.f. Mélikah ABDELMOUMEN, Liberté, féminité, fatalité, cyberentretien avec Nelly Arcan (en ligne), Spirale, ${ }^{\circ} 215,2007$, pp. 34-37.

16 Rappelons que dans le récit mythique, Pygmalion a pu épouser sa statue et que l'union heureuse de ce couple a donné deux enfants.

17Pierre BRUNEL, op. cit., p. 82.

18 Nous citons à titre d'exemple les deux premiers romans qui précédent $A C O$ : Putain (2001) et Folle (2004). Dans ces œuvres, les personnages masculins et féminins sont mis en scène par l'auteure à partir de la recréation des figures mythiques de Pygmalion et de Galatée ainsi que les mythèmes qui s'y rapportent. Ce qui nécessite en fait une étude étendue et approfondie qui dépassera les limites de notre travail. 